\title{
A WHITE DWARF MERGER AS PROGENITOR OF THE ANOMALOUS X-RAY PULSAR 4U 0142+61?
}

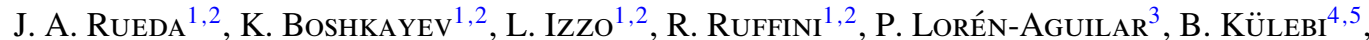 \\ G. AzNAR-Siguán ${ }^{5,6}$, AND E. GarCía-Berro ${ }^{5,6}$ \\ ${ }^{1}$ Dipartimento di Fisica and ICRA, Sapienza Università di Roma, P.le Aldo Moro 5, I-00185 Rome, Italy; jorge.rueda@icra.it \\ 2 ICRANet, P.zza della Repubblica 10, I-65122 Pescara, Italy \\ ${ }^{3}$ School of Physics, University of Exeter, Stocker Road, Exeter EX4 4QL, UK \\ ${ }^{4}$ Institut de Ciències de l'Espai (CSIC), Facultat de Ciències, Campus UAB, Torre C5-parell, E-08193 Bellaterra, Spain \\ ${ }^{5}$ Institute for Space Studies of Catalonia, c/Gran Capità 2-4, Edif. Nexus 104, E-08034 Barcelona, Spain; enrique.garcia-berro@upc.edu \\ ${ }^{6}$ Departament de Física Aplicada, Universitat Politècnica de Catalunya, c/Esteve Terrades, 5, E-08860 Castelldefels, Spain \\ Received 2013 March 1; accepted 2013 June 24; published 2013 July 16
}

\begin{abstract}
It has been recently proposed that massive, fast-rotating, highly magnetized white dwarfs could describe the observational properties of some of soft gamma-ray repeaters and anomalous X-ray pulsars (AXPs). Moreover, it has also been shown that high-field magnetic white dwarfs can be the outcome of white dwarf binary mergers. The products of these mergers consist of a hot central white dwarf surrounded by a rapidly rotating disk. Here we show that the merger of a double degenerate system can explain the characteristics of the peculiar AXP 4U 0142+61. This scenario accounts for the observed infrared excess. We also show that the observed properties of $4 \mathrm{U} 0142+6$ are consistent with an approximately $1.2 M_{\odot}$ white dwarf, remnant of the coalescence of an original system made of two white dwarfs of masses $0.6 M_{\odot}$ and $1.0 M_{\odot}$. Finally, we infer a post-merging age $\tau_{\mathrm{WD}} \approx 64 \mathrm{kyr}$ and a magnetic field $B \approx 2 \times 10^{8} \mathrm{G}$. Evidence for such a magnetic field may come from the possible detection of the electron cyclotron absorption feature observed between the $B$ and $V$ bands at $\approx 10^{15} \mathrm{~Hz}$ in the spectrum of $4 \mathrm{U} 0142+61$.
\end{abstract}

Key words: pulsars: general - pulsars: individual $(4 \mathrm{U} 0142+61)$ - stars: magnetic field - stars: rotation white dwarfs

\section{INTRODUCTION}

Soft gamma-ray repeaters (SGRs) are sources of short $(\approx 100 \mathrm{~ms})$, repeating bursts of soft $\gamma$-ray and X-ray radiation at irregular intervals, and share with anomalous X-ray pulsars (AXPs) several similarities, like rotation periods clustered between 2 and $12 \mathrm{~s}$, and high magnetic fields. Their observed spin-down rates range from $\dot{P} \approx\left(10^{-15}\right.$ to $\left.10^{-10}\right)$, and have typical X-ray luminosities in quiescent state $L_{X} \approx 10^{35} \mathrm{erg} \mathrm{s}^{-1}$. Currently, it is widely accepted that these objects are magnetars (Duncan \& Thompson 1992; Thompson \& Duncan 1995), although there are competing scenarios that challenge this model - see, for instance, the excellent and recent review by Mereghetti (2008) and references therein. Recently, Malheiro et al. (2012), following the pioneering works of Morini et al. (1988) and Paczynski (1990), have suggested an alternative model that could explain some properties of these sources. This model involves highly magnetized white dwarfs. For this model to be viable, the masses of the white dwarfs need to be rather large $\left(M \gtrsim 1.2 M_{\odot}\right)$, their magnetic fields should range from $B \approx 10^{7} \mathrm{G}$ all the way to $10^{10} \mathrm{G}$, and the rotation periods should be rather small, of the order of a few seconds. The most apparent drawback of this scenario, namely the rotational stability of fast-rotating white dwarfs, has been recently analyzed. Specifically, the crucial question of whether rotating white dwarfs can have rotation periods as short as the ones observed in AXPs has been recently addressed by Boshkayev et al. (2013b), who found that the minimum rotation period of typical carbon-oxygen white dwarfs is approximately $0.5 \mathrm{~s}$. Thus, since AXPs have rotation periods larger than this value they could be white dwarfs.

The existence of white dwarfs with magnetic fields ranging from $10^{7} \mathrm{G}$ up to $10^{9} \mathrm{G}$ is solidly confirmed by observations (Külebi et al. 2009). Observations show that most high-field magnetic white dwarfs (HFMWDs) are massive and, moreover, that none of them belongs to a non-interacting binary system, pointing toward a binary origin. However, although long-suspected (Wickramasinghe \& Ferrario 2000), it has only been recently shown that HFMWDs might be the result of white dwarf mergers. Smoothed particle hydrodynamics (SPH) simulations of the coalescence process indicate that the result of the merger is a white dwarf that contains the mass of the undisrupted primary, surrounded by a hot corona made of about half of the mass of the disrupted secondary. In addition, a rapidly rotating Keplerian disk which contains the rest of the material of the secondary is also formed, as little mass is ejected from the system during the coalescence process. The rapidly rotating hot corona is convective and an efficient $\alpha \omega$ dynamo can produce magnetic fields of up to $B \approx 10^{10} \mathrm{G}$ (García-Berro et al. 2012).

In view of these considerations, it is natural to ask ourselves if such binary mergers could also explain the properties of some AXPs. Here we explore such possibility for the specific case of the peculiar AXP 4U 0142+61. This AXP is by far the best observed source in the near-infrared (NIR), optical, and ultraviolet (UV) bands and has two characteristics that make it a peculiar object. The first is that $4 \mathrm{U} 0142+61$ presents a confirmed infrared excess (Hulleman et al. 2000) that might be attributed to an accretion disk, whereas the second one is that it is too bright for its cooling age, thus challenging the conventional magnetar model. Here we show that the properties of this AXP can be well explained by a model in which the central compact remnant is a massive magnetized white dwarf resulting from the merger of two otherwise ordinary white dwarfs, surrounded by the heavy accretion disk produced during the merger.

\section{A MODEL FOR 4 U $0142+61$}

To start with, we compute the approximate mass and radius of $4 \mathrm{U} 0142+61$. The stability of general relativistic uniformly rotating white dwarfs has been recently studied (Boshkayev et al. 2013b), and it has been shown that constant rotation 
Table 1

Bounds for the Mass, Radius, and Moment of Inertia of 4U 0142+61

\begin{tabular}{lcc}
\hline \hline & Minimum & Maximum \\
\hline$M\left(M_{\odot}\right)$ & 1.16 & 1.39 \\
$R_{\mathrm{eq}}\left(10^{8} \mathrm{~cm}\right)$ & 1.05 & 6.66 \\
$\langle R\rangle\left(10^{8} \mathrm{~cm}\right)$ & 1.05 & 6.03 \\
$I\left(\mathrm{~g} \mathrm{~cm}^{2}\right)$ & $2.9 \times 10^{48}$ & $1.4 \times 10^{50}$ \\
\hline
\end{tabular}

period sequences intersect the stability region of white dwarfs in two points that determine lower and upper bounds for the mass, equatorial/polar radii, and moment of inertia. In Table 1 we show the bounds for $4 \mathrm{U} 0142+61$. In this table $\langle R\rangle=\left(2 R_{\text {eq }}+R_{\mathrm{p}}\right) / 3$ denotes the mean-radius, where $R_{\text {eq }}$ and $R_{\mathrm{p}}$ are, respectively, the equatorial and polar radii.

\subsection{IR, Optical, and UV Photometry}

We next fitted the spectrum of $4 \mathrm{U} 0142+61$ as the sum of two components. The first is a blackbody:

$$
F_{\mathrm{BB}}=\pi \frac{2 h}{c^{2}}\left(\frac{R_{\mathrm{WD}}}{d}\right)^{2} \frac{v^{3}}{e^{h v /\left(k_{\mathrm{B}} T_{\mathrm{eff}}\right)}-1},
$$

where $R_{\mathrm{WD}}$ and $T_{\mathrm{eff}}$ are, respectively, the radius and effective temperature of the white dwarf. As it is shown below in Section 2.2, the system now behaves as an ejector, inhibiting the accretion of the disk material onto the central white dwarf. Thus, for the second component we adopted the blackbody disk model of Chiang \& Goldreich (1997), which is more appropriate for these systems (García-Berro et al. 2007):

$$
\begin{aligned}
F_{\mathrm{disk}}= & 12 \pi^{1 / 3} \cos i\left(\frac{R_{\mathrm{WD}}}{d}\right)^{2}\left(\frac{2 k_{\mathrm{B}} T_{\mathrm{eff}}}{3 h v}\right)^{8 / 3}\left(\frac{h v^{3}}{c^{2}}\right) \\
& \times \int_{x_{\mathrm{in}}}^{x_{\mathrm{out}}} \frac{x^{5 / 3}}{e^{x}-1} d x,
\end{aligned}
$$

where $i$ is the inclination angle of the disk, which we assume to be face-on, and $x=h v /\left(k_{\mathrm{B}} T\right)$. In this model the disk temperature $T$ varies as $r^{-3 / 4}$ (Chiang \& Goldreich 1997), with $r$ the distance from the center of the white dwarf. It is worth mentioning that in previous studies of 4U 0142+61 (Hulleman et al. 2000; Wang et al. 2006) the irradiated disk model of Vrtilek et al. (1990) has been used instead, but this model is more appropriate for accreting sources.

We computed the best fit of the spectrum parameters, finding $R_{\mathrm{WD}} \approx 0.006 R_{\odot}, T_{\mathrm{eff}} \approx 1.31 \times 10^{5} \mathrm{~K}$, inner and outer disk radii $R_{\text {in }}=0.97 R_{\odot}, R_{\text {out }}=51.1 R_{\odot}$ and correspondingly inner and outer disk temperatures $T_{\text {in }} \approx 1950 \mathrm{~K}$ and $T_{\text {out }} \approx 100 \mathrm{~K}$, respectively. In Figure 1 we show the photometric data of $4 \mathrm{U} 0142+61$ and our best-fit composite spectrum. The agreement of the composite spectrum with the observational data is quite good, taking into account that the high variability of the source in these bands can lead to changes in the optical fluxes of up to one order of magnitude (Durant \& van Kerkwijk 2006c). Thus, the white dwarf model is compatible with the observed photometry of $4 \mathrm{U} 0142+61$, as it seems to occur for SGR 0418+5729, Swift J1822.3-1606, and 1E 2259+586 (Boshkayev et al. 2013a).

To check whether this is a realistic and consistent model for $4 \mathrm{U} 0142+61$, we ran an SPH simulation of the merger of a $0.6+1.0 M_{\odot}$ binary white dwarf, which results in a central remnant of $\approx 1.1 M_{\odot}$, with a radius $R_{\mathrm{WD}} \approx 0.006 R_{\odot}$,

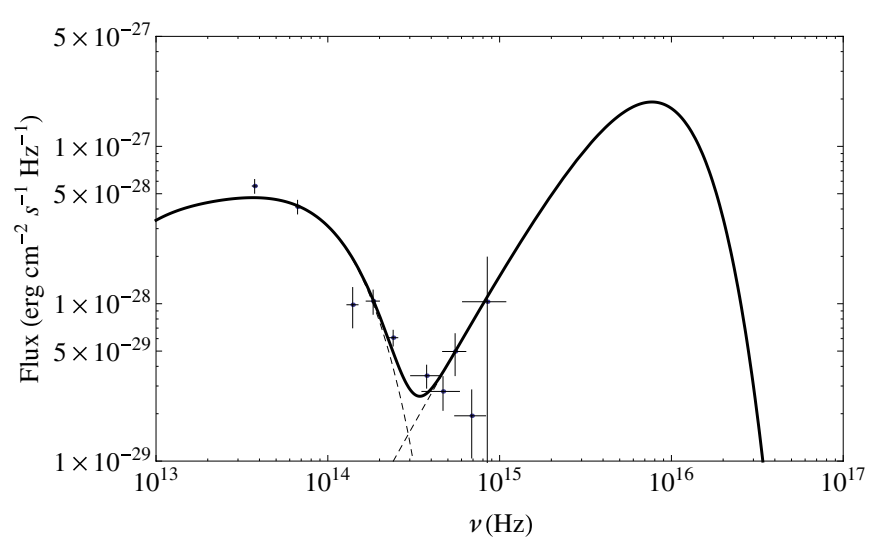

Figure 1. Observed and fitted spectrum of $4 \mathrm{U} 0142+61$. Due to the high variability of the source in the optical bands, we average all the existing data of the source in the different bands. All these data come from observations from 1994 October 31 up to 2005 July 26 (Hulleman et al. 2000, 2004; Dhillon et al. 2005; Morii et al. 2005, 2009; Durant \& van Kerkwijk 2006c). The result of the average is $V=25.66, R=25.25, I=23.76, J=22.04, H=20.70$, and $K=19.97$. There are upper limits in the $U$ and $B$ bands, $U=25.8$ (Dhillon et al. 2005) and $B=28.1$ (Hulleman et al. 2004), respectively. We also consider the observations of Wang et al. (2006) with the Spitzer/IRAC instrument at wavelengths $4.5 \mu \mathrm{m}$ and $8.0 \mu \mathrm{m}$. The fluxes are $36.3 \mu \mathrm{Jy}$ and $51.9 \mu \mathrm{Jy}$, respectively. We corrected the data for the interstellar extinction, using the estimated distance $d=3.6 \mathrm{kpc}$ (Durant \& van Kerkwijk 2006a) and an absorption in the $V$ band $A_{V}=3.5$ (Durant \& van Kerkwijk 2006b). For the rest of the bands we used $A_{U}=1.569 A_{V}, A_{B}=1.337 A_{V}, A_{R}=0.751 A_{V}$, $A_{I}=0.479 A_{V}, A_{J}=1.569 A_{V}, A_{J}=0.282 A_{V}, A_{H}=0.190 A_{V}$, and $A_{K}=0.114 A_{V}$ (Cardelli et al. 1989). The extinction in the Spitzer/IRAC bands for $A_{K}<0.5$ are $A_{4.5 \mu \mathrm{m}}=0.26 A_{K}$ and $A_{8.0 \mu \mathrm{m}}=0.21 A_{K}$ (Chapman et al. 2009).

in agreement with the photometric value. We recall that the central white dwarf accretes some material from the surrounding disk (of mass $M_{\text {disk }} \approx 0.5 M_{\odot}$ ) and thus shrinks a little. Moreover, the rotation period is $P \approx 15.7 \mathrm{~s}$ and the moment of inertia of the central white dwarf and the hot corona is $I \approx 2.0 \times 10^{50} \mathrm{~g} \mathrm{~cm}^{2}$, which is slightly larger than our maximum estimate-see Table 1. Furthermore, the magnetic field generated in the differentially rotating hot corona produced in the aftermath of the merger amounts to $B \approx 10^{10} \mathrm{G}$ (GarcíaBerro et al. 2012) which amply explains the magnetic field of 4U 0142+61; see Section 2.2.

\subsection{The Age and Magnetic Field of $4 U 0142+61$}

The presence of a disk around the magnetized white dwarf plays a key role in the evolution of its rotation period. This results from a delicate interplay between the interaction of the disk with the magnetosphere of the star, and accretion of disk matter onto the surface of the white dwarf. A solution of the magneto-hydrodynamic equations including the explicit coupling of magnetosphere-disk system and the mass and angular momentum transfer from the disk to the star is not yet available. For this reason the torque acting on the star it is often followed using a phenomenological treatment. We adopt the model of Armitage \& Clarke (1996), which assumes that the magnetic field lines threading the disk are closed. In this model the evolution of $\omega$ is dictated by

$$
\begin{aligned}
\dot{\omega}= & -\frac{2 B^{2}\langle R\rangle^{6} \omega^{3}}{3 I c^{3}} \sin ^{2} \theta+\frac{B^{2}\langle R\rangle^{6}}{3 I}\left[\frac{1}{R_{\mathrm{mag}}^{3}}-\frac{2}{\left(R_{\mathrm{c}} R_{\mathrm{mag}}\right)^{3 / 2}}\right] \\
& +\frac{\dot{M} R_{\mathrm{mag}}^{2} \omega}{I}
\end{aligned}
$$



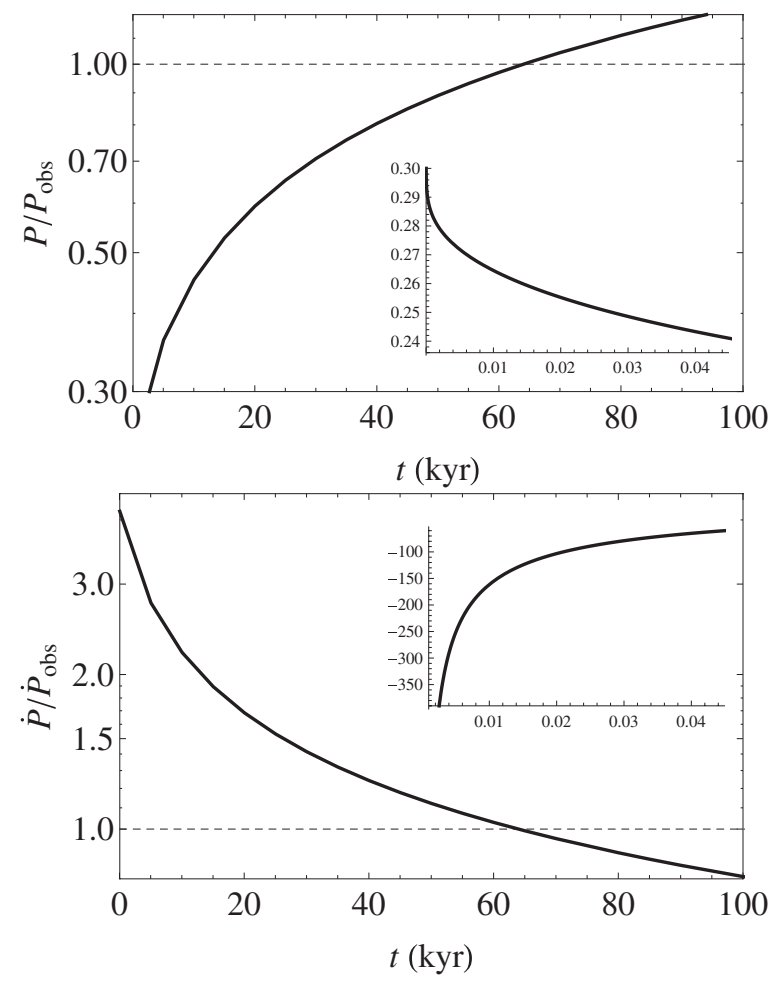

Figure 2. Time evolution of the period (top panel) and period derivative (bottom panel) of $4 \mathrm{U} 0142+61$. The insets show to the early evolutionary phases of the system.

where $\theta$ is the angle between the rotation axis and the magnetic dipole moment, $R_{\mathrm{mag}}=\left[B^{2}\langle R\rangle^{6} /(\dot{M} \sqrt{2 G M})\right]^{2 / 7}$ is the magnetospheric radius (Chatterjee et al. 2000; Toropina et al. 2012; Matt et al. 2012; Rueda \& Ruffini 2012), and $R_{\mathrm{c}}=\left(G M / \omega^{2}\right)^{1 / 3}$ is the corotation radius. The first term in Equation (3) describes the traditional magneto-dipole braking, the second one is the star-disk coupling, while the last one describes the angular momentum transfer from the disk to the white dwarf. We adopt an accretion rate corresponding to a Shakura-Sunyaev viscosity parameter $\alpha_{\mathrm{SS}}=0.1$ (Cannizzo et al. 1990; Chatterjee et al. 2000; Ertan et al. 2009). Adopting a misalignment angle $\theta=\pi / 2$ and integrating Equation (3) using the parameters resulting from our SPH simulation and it results that, for a wide range of magnetic field strengths, at early stages $R_{\text {mag }} \approx R_{\mathrm{WD}}$. Thus, initially the star is spun-up due to the large accretion rates-see the insets of Figure 2. However, after approximately $1 \mathrm{kyr}$, the inner radius of the disk-which is approximately given by the magnetospheric radius-becomes larger than the light cylinder radius, $R_{\mathrm{lc}}=c / \omega$. Hence, the disk cannot torque any longer the white dwarf, the rotation period reaches a minimum, and from this point on the disk and the star evolve independently, and accretion onto the magnetic poles stops. Thus, the star behaves as a normal pulsar, spinning-down by magneto-dipole radiation (Lamb et al. 1973; Chatterjee et al. 2000). The surface magnetic field needed to fit the observed values of $P$ and $\dot{P}$ when a mass $M=1.2 M_{\odot}$ is adopted is $B \approx 2.3 \times 10^{8} \mathrm{G}$ at an age $\tau_{\mathrm{sd}}=64 \mathrm{kyr}-$ see Figure 2. This age estimate compares well with the spin-down characteristic age $P /(2 \dot{P}) \approx 68$ kyr. Moreover, the strength of the magnetic field can be compared with that directly derived using the traditional misaligned dipole expression

$$
B=\sqrt{\frac{3 c^{3} I}{\langle R\rangle^{6}} \frac{P \dot{P}}{8 \pi^{2}}}
$$

(Ferrari \& Ruffini 1969; Lamb et al. 1973)—which in our case is valid because $R_{\text {in }} \approx R_{\odot}$ (see Section 2.1) is larger than the radius of the light cylinder $R_{\mathrm{lc}} \approx 0.6 R_{\odot}$. From the observed values $P=8.69$ s and $\dot{P}=2.03 \times 10^{-12}$ (Hulleman et al. 2000), we obtain $B=2.3 \times 10^{8} \mathrm{G}$ for $M_{\min }$, and $6.2 \times 10^{9} \mathrm{G}$ for $M_{\max }$, in agreement with the result obtained integrating Equation (3). Additionally, there are other indications that the magnetic field derived in this way is sound. In particular, the spectrum of 4U 0142+61 exhibits a significant drop-off between the $B$ and $V$ bands, at a frequency $v \approx 10^{15} \mathrm{~Hz}$; see Figure 1 . Hulleman et al. (2004) concluded that this feature is not due to variability and, moreover, they advanced that it is consistent with the electron cyclotron emission of a magnetic field $B \approx 10^{8} \mathrm{G}$. Adopting the minimum and maximum masses derived from our model, we obtain electron cyclotron frequencies $v_{\text {cyc }}=e B /\left(2 \pi m_{\mathrm{e}} c\right)=$ $6.3 \times 10^{14} \mathrm{~Hz}$ and $1.7 \times 10^{16} \mathrm{~Hz}$, which correspond to wavelengths that fall between the NIR and the UV, 0.5 and $176 \mu \mathrm{m}$, respectively. This suggests that the magnetic field must be closer to the lower value, and therefore that the corresponding mass should be approximately $1.2 M_{\odot}$. Actually, it is interesting to realize that although the mass of the remnant of the coalescence is slightly smaller than our fiducial mass for 4U $0142+61-$ $\approx 1.1$ and $\approx 1.2 M_{\odot}$, respectively - the mass accreted during the spin-up phase is $M_{\text {acc }} \approx 0.05 M_{\odot}$, in good agreement with the mass derived from the photometric solution. It could be argued that this is the maximum possible accreted mass, since during these early stages super-Eddington accretion rates are needed to accrete all the material inflowing from the disk. Nevertheless, during the very early stages after the merger the temperature of the coalesced system is very high, and the emission of neutrinos is not negligible (García-Berro et al. 2012).

We now compute the cooling age of $4 \mathrm{U} 0142+61$, and compare it with the spin-down age. As the hot, convective corona resulting from the merger is very short lived (García-Berro et al. 2012) the evolution of the surface luminosity of the white dwarf can be estimated using Mestel's cooling law (Mestel 1952):

$$
\tau_{\mathrm{cool}}=\frac{1}{\langle A\rangle}\left(\frac{b M Z^{2 / 5}}{L_{\mathrm{WD}}}\right)^{1 / x}-0.1
$$

where $\langle A\rangle$ is the average atomic weight of the core of the white dwarf, $Z$ is the metallicity of its envelope, $x=1.4, b=635$ (Hurley \& Shara 2003), and the rest of the symbols have their usual meaning. Adopting a carbon-oxygen core and $Z \approx 0.001$, which is a reasonable value (Althaus et al. 2010), we obtain a cooling age $\tau_{\text {cool }} \approx 64 \mathrm{kyr}$, in good agreement with the spin-down age.

\subsection{X-Ray Luminosity}

For a distance $d=3.6 \mathrm{kpc}$, Durant $\&$ van Kerkwijk (2006a) estimated an isotropic X-ray luminosity $L_{X}=4 \pi d^{2} f_{X}^{\text {unabs }} \approx$ $1.3 \times 10^{35} \mathrm{erg} \mathrm{s}^{-1}$, using the unabsorbed X-ray flux $f_{X}^{\text {unabs }}=$ $8.3 \times 10^{-11} \mathrm{erg} \mathrm{s}^{-1} \mathrm{~cm}^{-2}$ obtained by Patel et al. (2003). We use the result of the latest observations of $4 \mathrm{U} 0142+61$ with the EPIC cameras on board XMM-Newton, $f_{X}^{\text {unabs }}=$ $7.2 \times 10^{-11}$ erg s${ }^{-1} \mathrm{~cm}^{-2}$ (Göhler et al. 2005), obtaining $L_{X} \approx 1.1 \times 10^{35} \mathrm{erg} \mathrm{s}^{-1}$ when the same distance is adopted. The loss of rotational energy associated with the spin-down of $4 \mathrm{U}$ $0142+61, \dot{E}_{\text {rot }}=-4 \pi^{2} I \dot{P} / P^{3}$ gives $\left|\dot{E}_{\text {rot }}\right|=1.7 \times 10^{37} \mathrm{erg} \mathrm{s}^{-1}$ for $M_{\min }$ and $3.4 \times 10^{35} \mathrm{erg} \mathrm{s}^{-1}$ for $M_{\max }$, that cover the estimated $\mathrm{X}$-ray luminosity. 
The time-integrated X-ray spectrum of $4 \mathrm{U} 0142+61$ is well described by a blackbody and a power-law model with $k_{\mathrm{B}} T_{\mathrm{BB}}=$ $0.4 \mathrm{keV}$ and photon index $\Gamma=3.62$ (Göhler et al. 2005). The blackbody component corresponds to a temperature $T_{\mathrm{BB}} \approx$ $4.6 \times 10^{6} \mathrm{~K}$, which is higher than the surface temperature of a hot white dwarf. However, these systems may have coronal temperatures much higher than that of the surface (Malheiro et al. 2012), and thus the X-ray emission would be of magnetospheric origin. Because the inner radius of the disk is larger than the radius of the light cylinder $R_{\mathrm{lc}}$ (see Section 2.2), the mechanisms producing such radiation are similar to those of pulsars. In particular, a possible mechanism was delineated by Usov (1993), who showed that reheating of the magnetosphere by the bombardment of positrons moving backward to the surface of the star can produce large X-ray luminosities. Positrons are produced the interaction of highenergy photons with ultra-relativistic electrons, resulting in the creation of electron-positron pairs. Following closely the calculations of Usov (1993), we computed the theoretically expected X-ray luminosity of $4 \mathrm{U} 0142+61$. We found that the reheating of polar caps produces a persistent X-ray luminosity $L_{X} \approx 2 \times 10^{35} \mathrm{erg} \mathrm{s}{ }^{-1}$, in agreement with observations. Nonetheless, there are other possibilities. If the conventional magnetar interpretation is adopted, the X-ray luminosity would be due to the neutron star. Alternatively, it could also be due to ongoing accretion from a fossil disk onto the neutron star (Alpar 2001). In such cases the white dwarf product of the merger would have accreted enough material to undergo accretion-induced collapse to a neutron star.

\section{CONCLUSIONS}

We studied the possibility that the peculiar AXP 4U 0141+61 is a massive, fast-rotating, highly magnetized white dwarf, and we explored the viability of this object being the result of the coalescence of a binary white dwarf. Specifically, from its observed rotational velocity we first derived bounds for the mass, radius, and moment of inertia. Afterward, we fitted the IR, optical, and UV data of 4 U $0142+61$ with a composite spectrum made of two components, a blackbody and a dust disk, finding a good agreement with the observations. Moreover, we showed that the characteristics of the disk are consistent with the results of an SPH simulation of the merger of a $0.6+1.0 M_{\odot}$ binary system. We then estimated the age and the magnetic field of this AXP. Adopting the results of our SPH simulation we obtained a magnetic field $B=2.3 \times 10^{8} \mathrm{G}$, and a post-merger age $\approx 64 \mathrm{kyr}$. The cyclotron frequency of this magnetic field $v_{\text {cyc }} \approx 6 \times 10^{14} \mathrm{~Hz}$ would explain an absorption feature observed in the spectrum of $4 \mathrm{U} 0142+61$ at $v \approx 10^{15} \mathrm{~Hz}$. Furthermore, our age estimate is in excellent agreement with the white dwarf cooling age. We also showed that the X-ray luminosity of $4 \mathrm{U}$ $0142+61$ can be well explained by the rotational energy loss, and we inferred a theoretical estimate $L_{X} \approx 2 \times 10^{35} \mathrm{erg} \mathrm{s}^{-1}$, which agrees with the observed value, $L_{X} \approx 1.09 \times 10^{35} \mathrm{erg} \mathrm{s}^{-1}$. All these findings may support the hypothesis that the peculiar AXP 4U 0141+61 was originated in a white dwarf binary merger.

This work was partially supported by the MCINN grant AYA2011-23102, the AGAUR, the European Union FEDER funds, and the ESF EUROGENESIS project (grant EUI2009-04167).

\section{REFERENCES}

Alpar, M. A. 2001, ApJ, 554, 1245

Althaus, L. G., Córsico, A. H., Isern, J., \& García-Berro, E. 2010, A\&ARv, 18,471

Armitage, P. J., \& Clarke, C. J. 1996, MNRAS, 280, 458

Boshkayev, K., Izzo, L., Rueda, J. A., \& Ruffini, R. 2013a, A\&A, in press (arXiv:1305.5048)

Boshkayev, K., Rueda, J. A., Ruffini, R., \& Siutsou, I. 2013b, ApJ, 762, 117

Cannizzo, J. K., Lee, H. M., \& Goodman, J. 1990, ApJ, 351, 38

Cardelli, J. A., Clayton, G. C., \& Mathis, J. S. 1989, ApJ, 345, 245

Chapman, N. L., Mundy, L. G., Lai, S.-P., \& Evans, N. J., II. 2009, ApJ, 690, 496

Chatterjee, P., Hernquist, L., \& Narayan, R. 2000, ApJ, 534, 373

Chiang, E. I., \& Goldreich, P. 1997, ApJ, 490, 368

Dhillon, V. S., Marsh, T. R., Hulleman, F., et al. 2005, MNRAS, 363, 609

Duncan, R. C., \& Thompson, C. 1992, ApJL, 392, L9

Durant, M., \& van Kerkwijk, M. H. 2006a, ApJ, 650, 1070

Durant, M., \& van Kerkwijk, M. H. 2006b, ApJ, 650, 1082

Durant, M., \& van Kerkwijk, M. H. 2006c, ApJ, 652, 576

Ertan, Ü., Ekşi, K. Y., Erkut, M. H., \& Alpar, M. A. 2009, ApJ, 702, 1309

Ferrari, A., \& Ruffini, R. 1969, ApJL, 158, L71

García-Berro, E., Lorén-Aguilar, P., Aznar-Siguán, G., et al. 2012, ApJ, 749, 25

García-Berro, E., Lorén-Aguilar, P., Pedemonte, A. G., et al. 2007, ApJL, 661, L179

Göhler, E., Wilms, J., \& Staubert, R. 2005, A\&A, 433, 1079

Hulleman, F., van Kerkwijk, M. H., \& Kulkarni, S. R. 2000, Natur, 408, 689

Hulleman, F., van Kerkwijk, M. H., \& Kulkarni, S. R. 2004, A\&A, 416, 1037

Hurley, J. R., \& Shara, M. M. 2003, ApJ, 589, 179

Külebi, B., Jordan, S., Euchner, F., Gänsicke, B. T., \& Hirsch, H. 2009, A\&A, 506, 1341

Lamb, F. K., Pethick, C. J., \& Pines, D. 1973, ApJ, 184, 271

Malheiro, M., Rueda, J. A., \& Ruffini, R. 2012, PASJ, 64, 56

Matt, S. P., Pinzón, G., Greene, T. P., \& Pudritz, R. E. 2012, ApJ, 745, 101

Mereghetti, S. 2008, A\&ARv, 15, 225

Mestel, L. 1952, MNRAS, 112, 583

Morii, M., Kawai, N., Kataoka, J., et al. 2005, AdSpR, 35, 1177

Morii, M., Kobayashi, N., Kawai, N., et al. 2009, PASJ, 61, 51

Morini, M., Robba, N. R., Smith, A., \& van der Klis, M. 1988, ApJ, 333, 777

Paczynski, B. 1990, ApJL, 365, L9

Patel, S. K., Kouveliotou, C., Woods, P. M., et al. 2003, ApJ, 587, 367

Rueda, J. A., \& Ruffini, R. 2012, ApJL, 758, L7

Thompson, C., \& Duncan, R. C. 1995, MNRAS, 275, 255

Toropina, O. D., Romanova, M. M., \& Lovelace, R. V. E. 2012, MNRAS, 420,810

Usov, V. V. 1993, ApJ, 410, 761

Vrtilek, S. D., Raymond, J. C., Garcia, M. R., et al. 1990, A\&A, 235, 162

Wang, Z., Chakrabarty, D., \& Kaplan, D. L. 2006, Natur, 440, 772

Wickramasinghe, D. T., \& Ferrario, L. 2000, PASP, 112, 873 